Proceedings of the 2010 Winter Simulation Conference

B. Johansson, S. Jain, J. Montoya-Torres, J. Hugan, and E. Yücesan, eds.

\title{
REFLECTED VARIANCE ESTIMATORS FOR SIMULATION
}

\author{
Melike Meterelliyoz \\ Christos Alexopoulos \\ David Goldsman
}

H. Milton Stewart School of Industrial and Systems Engineering

Georgia Institute of Technology

Atlanta, GA 30332 USA

\begin{abstract}
We study reflected standardized time series (STS) estimators for the asymptotic variance parameter of a stationary stochastic process. These estimators are based on the concept of data re-use and allow us to obtain more information about the process with no additional sampling effort. Reflected STS estimators are computed from "reflections" of the original sample path. We show that it is possible to construct linear combinations of reflected estimators with smaller variance than the variance of each constituent estimator, often at no cost in bias. We provide Monte Carlo examples to show that the estimators perform as well in practice as advertised by the theory.
\end{abstract}

\section{INTRODUCTION}

A fundamental problem in simulation output analysis concerns the computation of point and confidence interval (CI) estimators for the mean, $\mu$, of a stationary discrete-time stochastic process $\left\{X_{j}: j=1,2, \ldots\right\}$. The point estimation of $\mu$ is an "easy" problem when the underlying system starts in steady state; the sample mean $\bar{X}_{n} \equiv n^{-1} \sum_{j=1}^{n} X_{j}$ based on the finite sample $X_{1}, \ldots, X_{n}$ is an unbiased estimator of $\mu$ and is usually the estimator of choice. In order to provide a measure of the sample mean's precision, an estimate of $\operatorname{Var}\left(\bar{X}_{n}\right)$ also needs to be calculated. If the $X_{j}$ 's are independent and identically distributed (i.i.d.) random variables, then the sample variance $S_{X}^{2}(n) \equiv(n-1)^{-1} \sum_{j=1}^{n}\left(X_{j}-\bar{X}_{n}\right)^{2}$ is an unbiased estimator of the population variance $\sigma_{X}^{2} \equiv \mathrm{E}\left[\left(X_{1}-\mu\right)^{2}\right]$. In this case, $\operatorname{Var}\left(\bar{X}_{n}\right)$ can be estimated by $S_{X}^{2}(n) / n$. By the Central Limit Theorem, an asymptotically (as $\left.n \rightarrow \infty\right)$ valid $100(1-\alpha) \%$ CI for $\mu$ is $\bar{X}_{n} \pm z_{1-\alpha / 2} S_{X}(n) / \sqrt{n}$, where $z_{\beta}$ is the $\beta$-quantile of the standard normal distribution.

Unfortunately, the $X_{j}$ 's are typically correlated in simulation problems. While $\bar{X}_{n}$ remains unbiased for $\mu, S_{X}^{2}(n)$ can be severely biased for $\sigma_{X}^{2}$. In addition, if the autocovariance function $R_{j} \equiv \operatorname{Cov}\left(X_{1}, X_{1+j}\right)$, for $j=0,1, \ldots$, is positive, it can be shown that $\mathrm{E}\left[S_{X}^{2}(n) / n\right] \ll \operatorname{Var}\left(\bar{X}_{n}\right)$ (Law 2007). In this case, valid CIs for $\mu$ can still be obtained based on estimators of the quantities $\left\{\sigma_{n}^{2} \equiv n \operatorname{Var}\left(\bar{X}_{n}\right): n=1,2, \ldots\right\}$ or their $\operatorname{limit}^{2} \equiv \lim _{n \rightarrow \infty} \sigma_{n}^{2}$, which is called the (asymptotic) variance parameter of the process.

One can find in the literature many techniques for estimating $\left\{\sigma_{n}^{2}: n=1,2, \ldots\right\}$ and $\sigma^{2}$, such as nonoverlapping batch means (NBM) (Schmeiser 1982), overlapping batch means (OBM) (Meketon and Schmeiser 1984), and standardized time series (STS) area (Schruben 1983) and Cramér-von Mises (CvM) (Goldsman, Kang, and Seila 1999) estimators. These techniques typically group observations into nonoverlapping or overlapping batches in order to produce low-variance estimators for the variance parameter.

Several techniques employ forms of data re-use in an effort to estimate $\sigma^{2}$. For instance, Foley and Goldsman (1999) consider orthonormal weight functions that yield a series of asymptotically i.i.d. STS area estimators based on a single data set. More-recent re-use methods include that of Calvin and Nakayama (2006), which averages multiple estimators based on permuted sample path segments, and that of Calvin (2007), which 


\section{Meterelliyoz, Alexopoulos, and Goldsman}

produces multiple estimators based on various iterated integrations of the sample path. Alexopoulos et al. (2010) and Meterelliyoz et al. (2009) develop folded area and CvM estimators based on nonoverlapping and overlapping batches. In addition, there is a substantial simulation literature on estimators incorporating jackknifing and/or bootstrapping; see, e.g., Batur, Goldsman, and Kim (2009).

This article makes the following contributions to variance estimation for steady-state simulations. First, it introduces a new class of variance parameter estimators employing data re-use, namely, estimators based on reflections of the STS formed by the entire sample. A reflection operation on the original STS yields another STS; both STSs converge (as the sample size goes to infinity) to Brownian bridges with a known cross-covariance structure. The second contribution is the development of linear combinations of reflected estimators with substantially smaller mean square error (MSE) than the constituent estimators.

The remainder of this article is organized as follows. Section 2 reviews background material, Section 3 defines and studies various reflected estimators, and Section 4 provides conclusions.

\section{BACKGROUND}

In this section, we give some assumptions, definitions, and results that are needed in the rest of this article. We start in $\$ 2.1$ with a list of necessary assumptions and a review of some basic properties regarding the convergence of stationary processes. We define the STS and some of the variance parameter estimators based on STS in $\$ 2.2$.

\subsection{Basics and Assumptions}

We start with a Functional Central Limit Theorem (FCLT) that holds for several classes of stationary processes, e.g., stationary strongly mixing processes, associated stationary processes, and regenerative processes; see, e.g., Glynn and Iglehart (1990).

Assumption FCLT Suppose that the series $\sigma^{2} \equiv \sum_{j=-\infty}^{\infty} R_{j}$ converges absolutely and $\sigma^{2}>0$. For each positive integer $n$, let

$$
Y_{n}(t) \equiv \frac{\lfloor n t\rfloor\left(\bar{X}_{\lfloor n t\rfloor}-\mu\right)}{\sigma \sqrt{n}}, \quad \text { for } t \in[0,1],
$$

where $\lfloor\cdot\rfloor$ is the greatest integer function and $\left\{X_{j}\right\}$ is stationary with mean $\mu$. Then

$$
Y_{n}(\cdot) \underset{n \rightarrow \infty}{\stackrel{\mathcal{D}}{\longrightarrow}} \mathcal{W}(\cdot)
$$

where $\mathcal{W}(\cdot)$ is a standard Brownian motion process on $[0,1]$, and $\underset{n \rightarrow \infty}{\stackrel{\mathcal{D}}{\rightarrow}}$ denotes weak convergence (as $n \rightarrow \infty)$ in the Skorohod space $D[0,1]$ of real-valued functions on $[0,1]$ that are right-continuous with left-hand limits (Billingsley 1968).

Assumptions A below refer to the output process and Assumptions F apply to weight functions used in STS estimators.

\section{Assumptions A}

1. The process $\left\{X_{j}: j \geq 1\right\}$ satisfies Assumption FCLT.

2. The autocovariance function decays exponentially, i.e., $\left|R_{j}\right|=O\left(\delta^{j}\right), j=1,2, \ldots$, for some $\delta \in(0,1)$.

\section{Assumptions F}

1. The function $f(\cdot)$ is normalized so that $\operatorname{Var}\left[\int_{0}^{1} f(t) \mathcal{B}(t) \mathrm{d} t\right]=1$, where $\mathcal{B}(\cdot)$ is a standard Brownian bridge process on $[0,1]$.

2. $f(t)$ is twice continuously differentiable in $[0,1]$.

3. $f(t)$ is symmetric about $t=1 / 2$; that is, $f(t)=f(1-t)$ for $t \in[0,1 / 2]$. 
The Brownian bridge process is defined by $\mathcal{B}(t)=t \mathcal{W}(1)-\mathcal{W}(t)$, for $t \in[0,1]$. All finite-dimensional distributions of $\mathcal{B}(\cdot)$ are normal with $\mathrm{E}[\mathcal{B}(t)]=0$ and $\operatorname{Cov}(\mathcal{B}(s), \mathcal{B}(t))=\min (s, t)-s t$ for $0 \leq s, t \leq 1$. One can also show $\mathcal{B}(\cdot)$ is independent of $\mathcal{W}(1)$.

In the rest of this article, we let $\gamma_{1} \equiv 2 \sum_{\ell=1}^{\infty} \ell R_{\ell}$. We use the "big-oh" notation $p(n)=O(q(n))$ to mean that there are positive constants $c$ and $k$ such that $0 \leq p(n) \leq c q(n)$ for all $n \geq k$, and we use the "little-oh" notation $p(n)=o(q(n))$ to mean that $\lim _{n \rightarrow \infty} p(n) / q(n)=0$. We also define the functions

$$
F(t) \equiv \int_{0}^{t} f(s) \mathrm{d} s \quad \text { and } \quad \bar{F}(t) \equiv \int_{0}^{t} F(s) \mathrm{d} s
$$

for $0 \leq t \leq 1$, and we let $F \equiv F(1)$ and $\bar{F} \equiv \bar{F}(1)$.

\subsection{Standardized Time Series Area Estimators}

In this section, we define the STS and review that STS area estimator for the variance parameter.

The STS based on the sample $X_{1}, \ldots, X_{n}$ is defined as

$$
T_{n}(t) \equiv \frac{\lfloor n t\rfloor\left(\bar{X}_{n}-\bar{X}_{\lfloor n t\rfloor}\right)}{\sigma \sqrt{n}}, \quad \text { for } t \in[0,1] .
$$

Under Assumptions A, it can be shown that (Schruben 1983)

$$
\left[\sqrt{n}\left(\bar{X}_{n}-\mu\right), \sigma T_{n}(\cdot)\right] \underset{n \rightarrow \infty}{\stackrel{\mathcal{D}}{\longrightarrow}}[\sigma \mathcal{W}(1), \sigma \mathcal{B}(\cdot)]
$$

This leads to the area estimator for the variance parameter.

Goldsman, Meketon, and Schruben (1990) introduced the weighted area estimator for $\sigma^{2}$, which is based on the square of the weighted area under the STS (2) and its limiting functional. These are defined by

$$
\mathcal{A}(f ; n) \equiv\left[\frac{1}{n} \sum_{j=1}^{n} f\left(\frac{j}{n}\right) \sigma T_{n}\left(\frac{j}{n}\right)\right]^{2} \quad \text { and } \quad \mathcal{A}(f) \equiv\left[\int_{0}^{1} f(t) \sigma \mathcal{B}(t) \mathrm{d} t\right]^{2}
$$

respectively. The weight function $f(\cdot)$ is assumed to satisfy Assumptions $\mathrm{F}$.

Under Assumptions A, the continuous mapping theorem (Billingsley 1968) implies that $\int_{0}^{1} f(t) \sigma \mathcal{B}(t) \mathrm{d} t \sim$ $\sigma N(0,1)$ and $\mathcal{A}(f ; n) \underset{n \rightarrow \infty}{\stackrel{\mathcal{D}}{\longrightarrow}} \mathcal{A}(f) \sim \sigma^{2} \chi_{1}^{2}$, where $N(0,1)$ denotes a standard normal random variable, and $\chi_{v}^{2}$ denotes a chi-squared random variable with $v$ degrees of freedom.

Under Assumptions A and F, Goldsman, Meketon, and Schruben (1990) show that the expected value of the weighted area estimator is

$$
\mathrm{E}[\mathcal{A}(f, n)]=\sigma^{2}-\frac{\left[(F-\bar{F})^{2}+\bar{F}^{2}\right] \gamma_{1}}{2 n}+O\left(1 / n^{2}\right)
$$

Further, if we assume that the sequence $\left\{\mathcal{A}^{2}(f ; n): n=1,2, \ldots\right\}$ is uniformly integrable (Billingsley 1968), then

$$
\lim _{n \rightarrow \infty} \operatorname{Var}[\mathcal{A}(f ; n)]=\operatorname{Var}[\mathcal{A}(f)]=2 \sigma^{4}
$$

Example 1. The most-basic area estimator from Schruben (1983) uses the constant weight function $f_{0}(t) \equiv \sqrt{12}$, $t \in[0,1]$. In this case, Equation (3) gives $\mathrm{E}\left[\mathcal{A}\left(f_{0} ; n\right)\right]=\sigma^{2}-3 \gamma_{1} / n+O\left(1 / n^{2}\right)$. We say that an estimator of $\sigma^{2}$ is first-order unbiased if it has bias of the form $o(1 / n)$. Goldsman, Meketon, and Schruben (1990) showed that the quadratic weight function $f_{2}(t) \equiv \sqrt{840}\left(3 t^{2}-3 t+1 / 2\right), t \in[0,1]$, results in a first-order unbiased estimator $\mathcal{A}\left(f_{2} ; n\right) . \triangleleft$ 


\section{REFLECTED ESTIMATORS}

\subsection{Basic Results on Reflected Sample Paths}

Assumptions A can be used to show that certain functionals of a stationary process converge in distribution to the associated functionals of a Brownian motion process. In this section, we take advantage of the reflection principle of Brownian motion to produce new estimators for $\sigma^{2}$.

Reflection Principle: If $\mathcal{W}(t)$ is a Brownian motion on [0,1], then

$$
\mathcal{W}_{c}(t) \equiv \begin{cases}\mathcal{W}(t) & \text { if } 0 \leq t<c \\ 2 \mathcal{W}(c)-\mathcal{W}(t) & \text { if } c \leq t \leq 1\end{cases}
$$

is also a Brownian motion process, where $c \in[0,1]$ is any reflection point. Note that the processes $\mathcal{W}(\cdot)$ and $\mathcal{W}_{c}(\cdot)$ are correlated.

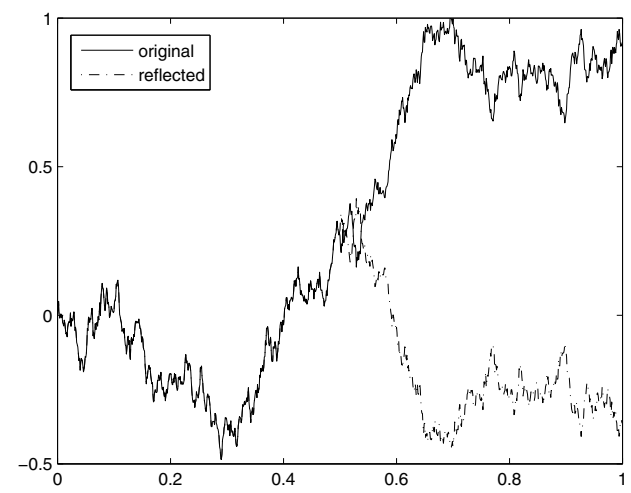

Figure 1: Original and Reflected Brownian Motion Processes $(c=0.5)$

Figure 1 shows an example of the original and reflected Brownian motion processes corresponding to the reflection point $c=0.5$. The reflection principle tells us that Brownian motion processes reflected after they hit any point $c \in[0,1]$ preserve the same distributional properties as the original Brownian motion. Hence, we can use a sample path from a Brownian motion process to generate several other different sample paths from the same process. This suggests that a single set of data from a simulation output process can be re-used to obtain alternative sample data sets, where their respective sample functionals $Y_{n}(t)$ still converge in distribution to the corresponding Brownian motion processes. Not surprisingly, the new sample paths generated through reflection are generally correlated - and this correlation is taken into account in the subsequent analysis.

We will henceforth assume that the mean $\mu$ for this process is zero. For processes with $\mu \neq 0$, we can consider (without loss of generality) the difference of two independent realizations, so that the mean and the variance of the sample mean of the difference are zero and approximately $2 \sigma^{2} / n$, respectively. Hence the assumption of $\mu=0$ is legitimate. In any case, define

$$
X_{c, j} \equiv \begin{cases}X_{j} & \text { if } 1 \leq j \leq\lfloor c n\rfloor \\ -X_{j} & \text { if }\lfloor c n\rfloor+1 \leq j \leq n\end{cases}
$$

for $j=1, \ldots, n$. Let $S_{c, k} \equiv \sum_{j=1}^{k} X_{c, j}$ for $k=1,2, \ldots$ One has

$$
S_{c,\lfloor n t\rfloor} \equiv \begin{cases}S_{\lfloor n t\rfloor} & \text { if } 0 \leq t<c \\ 2 S_{\lfloor n c\rfloor}-S_{\lfloor n t\rfloor} & \text { if } c \leq t \leq 1\end{cases}
$$


Further define $X_{c, n}(t) \equiv S_{c,\lfloor n t\rfloor} /(\sigma \sqrt{n})$, and note that, by Equation (1) with $\mu=0$,

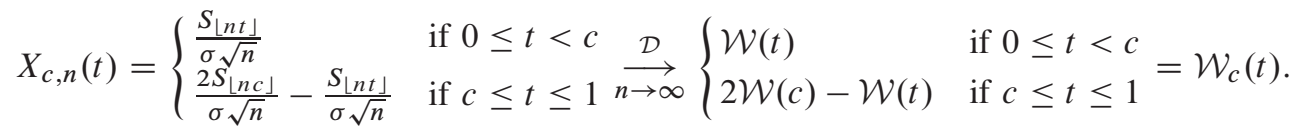

Thus, we have shown that the process $X_{c, n}(\cdot)$ obtained from the reflected data $\left\{X_{c, j}: j=1, \ldots, n\right\}$ converges to the limiting Brownian motion that results from a reflection at point $\lfloor c n\rfloor$ of the original process $\left\{X_{j}: j=1, \ldots, n\right\}$.

In $\$ 2$, we reviewed estimators based on the $\operatorname{STS} T_{n}(\cdot)$ and showed that their limiting functionals are based on functionals of Brownian bridge processes. For $t \in[0,1]$, the reflected STS with reflection point $c$ is

$$
T_{c, n}(t) \equiv \frac{\lfloor n t\rfloor\left(\bar{X}_{c, n}-\bar{X}_{c,\lfloor n t\rfloor}\right)}{\sigma \sqrt{n}}=\frac{t S_{c, n}}{\sigma \sqrt{n}}-\frac{S_{c,\lfloor n t\rfloor}}{\sigma \sqrt{n}}-\frac{(n t-\lfloor n t\rfloor) \bar{X}_{c, n}}{\sigma \sqrt{n}},
$$

where $\bar{X}_{c, n} \equiv \frac{1}{n} \sum_{j=1}^{n} X_{c, j}$. Analogously to a standard Brownian bridge $\mathcal{B}(t)$, the reflected Brownian bridge process with reflection point $c$ can be expressed as

$$
\mathcal{B}_{c}(t) \equiv t \mathcal{W}_{c}(1)-\mathcal{W}_{c}(t)= \begin{cases}t[2 \mathcal{W}(c)-\mathcal{W}(1)]-\mathcal{W}(t) & \text { if } 0 \leq t<c \\ \mathcal{W}(t)+2(t-1) \mathcal{W}(c)-t \mathcal{W}(1) & \text { if } c \leq t \leq 1\end{cases}
$$

It is easy to show that $\operatorname{Cov}\left(\mathcal{B}_{c}(s), \mathcal{B}_{c}(t)\right)=\min (s, t)-s t$ for $0 \leq s, t \leq 1$, and hence $\mathcal{B}_{c}(t)$ is indeed a Brownian bridge process. Equation (4) and Assumptions A imply $T_{c, n}(t) \underset{n \rightarrow \infty}{\stackrel{\mathcal{D}}{\longrightarrow}} \mathcal{B}_{c}(t)$ since

$$
\frac{(n t-\lfloor n t\rfloor) \bar{X}_{c, n}}{\sigma \sqrt{n}} \stackrel{\mathcal{P}}{\longrightarrow} 0
$$

where $\stackrel{\mathcal{P}}{\longrightarrow}$ denotes convergence in probability as $n \rightarrow \infty$.

\subsection{Reflected Area Estimators}

We define the reflected area estimator $\mathcal{A}_{c}(f ; n)$ with reflection point $c \in[0,1]$ and its limiting functional $\mathcal{A}_{c}(f)$ as

$$
\mathcal{A}_{c}(f ; n) \equiv\left[\frac{1}{n} \sum_{j=1}^{n} f\left(\frac{j}{n}\right) \sigma T_{c, n}\left(\frac{j}{n}\right)\right]^{2} \quad \text { and } \quad \mathcal{A}_{c}(f) \equiv\left[\int_{0}^{1} f(t) \sigma \mathcal{B}_{c}(t) \mathrm{d} t\right]^{2}
$$

respectively, where $f(\cdot)$ is a weight function satisfying Assumptions F. Under Assumptions A, it can be shown that $\mathcal{A}_{c}(f ; n) \underset{n \rightarrow \infty}{\stackrel{\mathcal{D}}{\longrightarrow}} \mathcal{A}_{c}(f)$. The following theorem gives the expected values of $\mathcal{A}_{c}(f ; n)$ for the weight functions $f_{0}(\cdot)$ and $f_{2}(\cdot)$. For notational convenience, we assume $c n$ to be integer. The proof can be found in Meterelliyoz (2008).

Theorem 1. If $c \in[0,1]$ and Assumptions $A$ and $F$ hold, then

$$
\mathrm{E}\left[\mathcal{A}_{c}\left(f_{0}, n\right)\right]=\sigma^{2}-\left(24 c^{2}-24 c+3\right) \frac{\gamma_{1}}{n}+O\left(1 / n^{2}\right)
$$

and

$$
\mathrm{E}\left[\mathcal{A}_{c}\left(f_{2} ; n\right)\right]=\sigma^{2}-420\left(4 c^{6}-12 c^{5}+13 c^{4}-6 c^{3}+c^{2}\right) \frac{\gamma_{1}}{n}+O\left(1 / n^{2}\right)
$$

Remark 1. Note that the first-order bias term in Equation (5) vanishes when $c=0.14645$ and 0.85355. Similarly, $c=0,1 / 2$, and 1 eliminates the first-order bias in Equation (6). In particular, reflection in the middle of the sample yields a first-order unbiased estimator for the weight function $f_{2}$. 


\subsubsection{Linear Combinations of Reflected Area Estimators}

We can obtain more-precise estimators in terms of variance if we find appropriate linear combinations of the original and reflected estimators. This is also consistent with the principle of data re-use. To this end, we consider estimators of the form $\sum_{j=1}^{k} \alpha_{j} \mathcal{A}_{c_{j}}(f ; n)$, where $0 \leq c_{1} \leq c_{2} \leq \cdots \leq c_{k} \leq 1$ are the reflection points and we aim at minimizing the variance of the limiting functional $\sum_{j=1}^{k} \alpha_{j} \mathcal{A}_{c_{j}}(f)$. If $\boldsymbol{\alpha} \equiv\left(\alpha_{1}, \alpha_{2}, \ldots, \alpha_{k}\right)$ and $c \equiv\left(c_{1}, c_{2}, \ldots, c_{k}\right)$, the underlying optimization problem can be stated formally as

$$
\begin{array}{cl}
\min _{\boldsymbol{\alpha}, \boldsymbol{c}} & \operatorname{Var}\left[\sum_{j=1}^{k} \alpha_{j} \mathcal{A}_{c_{j}}(f)\right] \\
\text { subject to } & \sum_{j=1}^{k} \alpha_{j}=1 \\
& 0 \leq c_{1} \leq c_{2} \leq \ldots \leq c_{k} \leq 1 \\
& \alpha_{j} \in \mathbb{R} ; j=1, \ldots, k .
\end{array}
$$

While this linear combination has minimum asymptotic variance, it is also asymptotically unbiased. Meterelliyoz (2008) provides the detailed solution of Problem (7), along with expressions for the optimal variance for various weights $f$ and values of $k$.

Example 2. For weight function $f_{0}$, the optimal reflection points and weight combinations for two linearly combined reflected area estimators are $\{0,0.5\}$ and $\{0.5,0.5\}$, respectively. If we linearly combine three estimators, the optimal values will be $\{0,0.153,0.847\}$ and $\{0.33,0.33,0.33\}$, respectively.

If we solve the Problem (7) for weight function $f_{2}$ with $k=2$, the optimal reflection points and weight combinations will be $\{0,0.5\}$ and $\{0.5,0.5\}$, respectively. For $k=3$, the solution is $\{0.208,0.65,0.86\}$ and $\{0.33,0.33,0.33\}$, respectively.

Example 3. Using the optimal reflection points and weight combinations from Example 2, we obtain the respective expected values and variances of variance parameter estimators for a first-order autoregressive $(\mathrm{AR}(1))$ process with $\phi=0.9$. An $\mathrm{AR}(1)$ process is a stationary (Gaussian) process that is defined by $X_{i}=\phi X_{i-1}+\epsilon_{i}$ for $i=1,2, \ldots$, where $1<\phi<1, X_{0} \sim N(0,1)$, and the $\epsilon_{i}$ 's are i.i.d. $N\left(0,1-\phi^{2}\right)$ random variables that are independent of $X_{0}$. This process has covariance function $R_{k}=\phi^{|k|}$, for $k=0,1,2, \ldots$, so that $\sigma^{2}=(1+\phi) /(1-\phi)$ and $\gamma_{1}=2 \phi /(1-\phi)^{2}$. In Table 1 , theoretical and simulated results using $n=50000$ observations are listed; all theoretical values are derived in Meterelliyoz (2008). We see that the point estimates are very close to the theoretical values, indicating that the estimators perform as advertised. In particular, we see that the asymptotic variance of the area estimator for one long run (batch) of observations for the AR(1) process under study is $2 \sigma^{4}=722$. Further, the combination of merely two reflected estimators induces a reduction in variance of about $50 \%$, while the combination of $k=3$ estimators yields an additional reduction of about $10 \%$.

\section{CONCLUSIONS}

This article studied estimators for the asymptotic variance parameter of a stationary stochastic process. The idea is to form optimal linear combinations of estimators obtained from various reflections of the original (entire) sample path. First of all, we considered the reflected version of a standardized time series area estimator based on an arbitrary reflection point. The optimal reflection points and coefficients of the linearly combined area estimators were obtained by solving (nonconvex) optimization problems. These linear combinations yielded estimators with significantly smaller variances compared to those of the individual estimators. To complement the theoretical work, we conducted Monte Carlo experiments, which confirmed our theoretical results. In future work, we will obtain confidence intervals for $\sigma^{2}$ and $\mu$ using the reflected estimators. We will also study analogous estimators based on STS Cramér-von Mises estimators as well as estimators incorporating batching. In addition, we will combine folding and reflection principles to obtain even-better estimators of the variance parameter. 
Table 1: Theoretical and Estimated Means and Variances of Linearly Combined Reflected Area Estimators for an AR(1) Process with $\phi=0.9$

\begin{tabular}{|c|c|cc|cc|}
\hline Weight & $k$ & Theoretical Mean & Estimated Mean & Theoretical Variance & Estimated Variance \\
\hline \multirow{3}{*}{$f_{0}$} & 1 & 18.95 & 19.00 & 722.00 & 724.20 \\
& 2 & 19.00 & 18.98 & 361.00 & 362.77 \\
& 3 & 18.98 & 18.98 & 294.15 & 293.60 \\
\hline \multirow{3}{*}{$f_{2}$} & 1 & 19.00 & 18.97 & 722.00 & 718.26 \\
& 2 & 19.00 & 18.97 & 361.00 & 359.80 \\
& 3 & 18.94 & 18.96 & 293.56 & 293.27 \\
\hline
\end{tabular}

\section{REFERENCES}

Alexopoulos, C., C. F. Antonini, D. Goldsman, and M. Meterelliyoz. 2010. Performance of folded variance estimators for simulation. To appear in ACM Transactions on Modeling and Computer Simulation.

Batur, D., D. Goldsman, and S.-H. Kim. 2009. An improved standardized time series Durbin-Watson variance estimator for steady-state simulation. Operations Research Letters 37:285-289.

Billingsley, P. 1968. Convergence of probability measures. New York: John Wiley \& Sons.

Calvin, J. M. 2007. Simulation output analysis using integrated paths. ACM Transactions on Modeling and Computer Simulation 17 (3).

Calvin, J. M., and M. K. Nakayama. 2006. Permuted standardized time series for steady-state simulations. Mathematics of Operations Research 31:351-368.

Foley, R., and D. Goldsman. 1999. Confidence intervals using orthonormally weighted standardized time series. ACM Transactions on Modeling and Computer Simulation 9:297-325.

Glynn, P. W., and D. Iglehart. 1990. Simulation analysis using standardized time series. Mathematics of Operations Research 15:1-16.

Goldsman, D., K. Kang, and A. F. Seila. 1999. Cramér-von Mises variance estimators for simulations. Operations Research 47:299-309.

Goldsman, D., M. Meketon, and L. Schruben. 1990. Properties of standardized time series weighted area variance estimators. Management Science 36:602-616.

Law, A. M. 2007. Simulation modeling and analysis. 4th ed. New York: McGraw-Hill.

Meketon, M. S., and B. W. Schmeiser. 1984. Overlapping batch means: Something for nothing? In Proceedings of the 1984 Winter Simulation Conference, ed. S. Sheppard, U. W. Pooch, and C. D. Pedgen, 227-230. Piscataway, NJ: Institute of Electrical and Electronics Engineers.

Meterelliyoz, M. 2008. Variance Parameter Estimation Methods with Re-use of Data. Ph. D. thesis, H. Milton Stewart School of Industrial and Systems Engineering, Georgia Institute of Technology, Atlanta, GA.

Meterelliyoz, M., C. Alexopoulos, and D. Goldsman. 2009. Folded overlapping variance estimators for simulation. Technical report, H. Milton Stewart School of Industrial and Systems Engineering, Georgia Institute of Technology, Atlanta, GA.

Schmeiser, B. W. 1982. Batch size effects in the analysis of simulation output. Operations Research 30:556-568. Schruben, L. 1983. Confidence interval estimation using standardized time series. Operations Research 31:1167-1178.

\section{AUTHOR BIOGRAPHIES}

MELIKE METERELLIYOZ recently completed a Postdoctoral fellowship in the College of Management at the Georgia Institute of Technology. She holds a Ph.D. from the H. Milton Stewart School of Industrial and Systems Engineering at the Georgia Institute of Technology. Her research interests are in the areas of simulation output analysis, statistics, revenue management and discrete choice modeling. She is a member of INFORMS and an active participant in the Winter Simulation Conference. Her e-mail address is melike@gatech . edu. 
CHRISTOS ALEXOPOULOS is an Associate Professor in The H. Milton Stewart School of Industrial and Systems Engineering at the Georgia Institute of Technology. His research interests are in the areas of simulation, statistics, and optimization of stochastic systems. He is a member of INFORMS and an active participant in the Winter Simulation Conference, having been Proceedings Co-Editor in 1995, Associate Program Chair in 2006, and a member of the Board of Directors since 2008. He is also an Area Editor of the ACM Transactions on Modeling and Computer Simulation. His e-mail address is christos@isye.gatech.edu, and his web page is ww. isye.gatech. edu/ christos.

DAVID GOLDSMAN is a Professor in the H. Milton Stewart School of Industrial and Systems Engineering at the Georgia Institute of Technology. His research interests include simulation output analysis, ranking and selection, and healthcare simulation. He was Program Chair of the Winter Simulation Conference in 1995, and he served on the WSC Board of Directors from 2002 to 2010. His e-mail address is sman@isye.gatech.edu, and his web page is www. isye.gatech.edu/ $\sim \operatorname{sman}$. 